\title{
Quality improvement for reducing intravenous patient-controlled analgesia self-discontinuation rate
}

\author{
Yoo-Jin Kang ${ }^{1}$, Seong-Joo Eun², Eun-Woo Park ${ }^{2}$, Soo-Young Lee ${ }^{2}$, and Jong-Man Kang ${ }^{2}$ \\ Department of Anesthesiology and Pain Medicine, ${ }^{1}$ Kyung Hee University Hospital, Kyung Hee University School of Medicine, \\ ${ }^{2}$ Kyung Hee University Hospital at Gangdong, Kyung Hee University, Seoul, Korea
}

Hospitals have undertaken quality improvement (QI) efforts to address patient care deficiencies and improve hospital systems. Identifying system defects and devising better ways to do things can be challenging. An effective QI program can improve clinical activity [1]. Many hospitals are actively engaged in improvement efforts, but these activities vary in method and impact. Hospitals follow trends that set QI as a new strategic priority, employ quality practices consistent with aims, strengthen staff training and involvement in QI methods, and engage an array of QI activities and clinical QI strategies.

Postoperative pain is an important complication encountered after surgery. With its capacity for self-adjustability, intravenous patient-controlled analgesia (IV-PCA) use in controlling postoperative pain has become widespread [2]. This can reduce hospitalization time by facilitating recovery and promoting wound healing. However, discontinuing IV-PCA due to any side effects leads to patient dissatisfaction and resource waste.

In our hospital, each health care system unit makes an effort to improve its processes in cooperation with the QI department. The anesthesia and post-anesthesia care unit nurse team focused on PCA problems, and planned to improve patient PCA satisfaction in response to QI.

Two hundred and forty nine patients, ASA I or II, aged 20-60 years, with no history of fentanyl and ondansetron side effects who had IV-PCA after abdominal general surgery, were included in this study. Patients supplied informed consent. Patients with major organ diseases, or who were pregnant or allergic to fentanyl or ondansetron, were excluded.

Patients did not receive premedication. General anesthesia was induced with propofol $1.5-2 \mathrm{mg} / \mathrm{kg}$ and rocuronium 0.8 $\mathrm{mg} / \mathrm{kg}$, and maintained with sevoflurane $1.5-3$ vol\%, $\mathrm{O}_{2}-\mathrm{N}_{2} \mathrm{O}$ $3 \mathrm{~L} / \mathrm{min}\left(\mathrm{FIO}_{2} \mathrm{0.5}\right)$. Standard monitoring (ECG, non-invasive blood pressure and pulse oximetry) was established during anesthesia. When surgery was finished, muscle relaxation was reversed by glycopyrrolate with pyridostigmine administration. Other antiemetics were not used during and after anesthesia, except for ondansetron mixed in the IV-PCA. The IV-PCA basal rate was $2 \mathrm{ml} / \mathrm{h}$, bolus injection was $2 \mathrm{ml}$, lockout time was set to $15 \mathrm{~min}$. IV-PCA was connected $10 \mathrm{~min}$ before the end of surgery.

In the first survey, all the patients had IV-PCA, including fentanyl $1,500 \mu \mathrm{g}$ and ondansetron $4 \mathrm{mg}$ with normal saline (total $100 \mathrm{ml}$ ), regardless of patient body weight or surgery type. Discontinuation after PCA was $20.5 \%$. The reasons for removing IV-PCA were postoperative nausea and vomiting (PONV) (64.7\%), pain resolution (11.7\%), dizziness (11.7\%), and early discharge (1.9\%).

The QI anesthesia nurse team worked with anesthesiologists and took several steps to decrease PCA discontinuation rate. First, fentanyl dose was planned according to surgery type and patient body weight to reduce side effects: $15-20 \mu \mathrm{g} / \mathrm{kg}$ for major surgery (ex. gastrectomy) and 10-15 $\mu \mathrm{g} / \mathrm{kg}$ for minor surgery (ex. appendectomy, laparoscopic cholecystectomy). Second, ondansetron was increased from $4 \mathrm{mg}$ to $12 \mathrm{mg}$. Third, patients were educated more intensively to convey that IV-PCA is not a complete solution to pain relief, but that it can largely reduce pain. They were also informed about possible side effects and their management.

Corresponding author: Jong-Man Kang, M.D., Ph.D., Department of Anesthesiology and Pain Medicine, Kyung Hee University Hospital at Gangdong, 892, Dongnam-ro, Gangdong-gu, Seoul 134-090, Korea. Tel: 82-2-440-6193, Fax: 82-2-440-7808, E-mail: jongmankang@gmail.com (c) This is an open-access article distributed under the terms of the Creative Commons Attribution Non-Commercial License (http:// creativecommons.org/licenses/by-nc/3.0/), which permits unrestricted non-commercial use, distribution, and reproduction in any medium, provided the original work is properly cited. 
Table 1. Causes for Discontinuation of Intravenous Patient-controlled Analgesia

\begin{tabular}{lccc}
\hline $\begin{array}{c}\text { Discontinuation } \\
\text { causes }\end{array}$ & $\begin{array}{c}\text { pre-QI group } \\
(\mathrm{n}=51)\end{array}$ & $\begin{array}{c}\text { post-QI group } \\
(\mathrm{n}=26)\end{array}$ & P value \\
\hline PONV & $33(64.7)$ & $10(38.5)$ & 0.033 \\
Resolved pain & $6(11.7)$ & $7(26.9)$ & 0.114 \\
Dizziness & $6(11.7)$ & $5(19.2)$ & 0.493 \\
Early discharge & $1(1.9)$ & $2(7.7)$ & 0.262 \\
Other & $5(9.8)$ & $2(7.7)$ & 1.000 \\
\hline
\end{tabular}

Data are presented as the number of patients (\%). QI: Quality Improvement, PONV: postoperative nausea and vomiting.

A month later, we investigated 224 patients under the same conditions and compared discontinuation rates after PCA, and the reasons for discontinuation between pre-QI and post-QI groups. Data were expressed as number of patients (\%). Statistical analysis was conducted using SPSS for Windows version 12.0 (SPSS, Chicago, IL, USA). Variables between groups were compared using Fisher's exact test. A P value $<0.05$ was considered statistically significant.

The post-QI group IV-PCA discontinuance rate was down to $11.6 \%$. The PONV rate was down to $38.5 \%$ (Table 1 ). Resolved pain, dizziness, and early discharge rates were increased in the post-QI group, but absolute occurrence numbers were similar between groups.

QI usually starts with healthcare system retrospective structure and process reviews. It can be a great opportunity to "think outside the box." Hospitals are engaging in QI activities in order to deliver high quality patient care. QI has become a priority in developing new strategies and plans for hospitals.

Opioid-based IV-PCA is regarded as a safe method for controlling patient postoperative pain [3]. However, it is frequently discontinued due to many kinds of discomfort. PONV was one of the most common causes. Therefore, we focused mainly on preventing PONV. Due to common patient discomfort caused by PONV, this has been investigated in many studies, and many new drugs have been recently developed [4]. A 5-hydroxytryptamine receptor antagonist is generally used, because they effectively treat and prevent PONV, and have few side effects [1]. Ondansetron is a widely used 5-hydroxytryptamine receptor antagonist [5]. The dose response relationship is well-known for late outcomes, and 1-16 mg intravenous ondansetron is commonly used.

We demonstrated that the outcome of PCA was improved by opioid dose adjustment according to patient weight and antiemetic dosage. This study may seem to be very simple and of little value from an academic perspective, but it is meaningful because it was performed by a nurse anesthesia team and postanesthesia care unit with anesthesiologist input and showed that a small effort can change the results of clinical activity. Patients, nurses, doctors and other health providers should communicate and work together to improve hospital care delivery.

In conclusion, simple QI activity reduced PCA discontinuation rates and side effects via drug dose adjustment. Furthermore, it saved medical resources and increased patient satisfaction with IV-PCA use. QI activities should be encouraged in hospitals.

\section{References}

1. Cohen AB, Restuccia JD, Shwartz M, Drake JE, Kang R, Kralovec P, et al. A survey of hospital quality improvement activities. Med Care Res Rev 2008; 65: 571-95.

2. Kim YY, Moon SY, Song DU, Lee KH, Song JW, Kwon YE. Comparison of palonosetron with ondansetron in prevention of postoperative nausea and vomiting in patients receiving intravenous patient-controlled analgesia after gynecological laparoscopic surgery. Korean $J$ Anesthesiol 2013; 64: 122-6.

3. Na HS, An SB, Park HP, Lim YJ, Hwang JW, Jeon YT, et al. Intravenous patient-controlled analgesia to manage the postoperative pain in patients undergoing craniotomy. Korean J Anesthesiol 2011; 60: 30-5.

4. Lee SJ, Lee SM, Kim SI, Ok SY, Kim SH, Park SY, et al. The effect of aprepitant for the prevention of postoperative nausea and vomiting in patients undergoing gynecologic surgery with intravenous patient controlled analgesia using fentanyl: aprepitant plus ramosetron vs ramosetron alone. Korean J Anesthesiol 2012; 63: 221-6.

5. Choi DK, Chin JH, Lee EH, Lim OB, Chung CH, Ro YJ, et al. Prophylactic control of post-operative nausea and vomiting using ondansetron and ramosetron after cardiac surgery. Acta Anaesthesiol Scand 2010; 54: 962-9. 